Article

\title{
Analysis of the Psychometric Properties of Perceived Motivational Climate in Sport Questionnaire and Its Relationship to Physical Activity and Gender Using Structural Equation Modelling
}

\author{
Félix Zurita Ortega ${ }^{1}$, Manuel Castro Sánchez ${ }^{2}$, Ramón Chacón Cuberos ${ }^{3, *}$, \\ Javier Cachón Zagalaz ${ }^{4}$, Cristian Cofré Bolados ${ }^{5,6}$, Emily Knox ${ }^{7}$ (i) and José Joaquín Muros ${ }^{1}$ \\ 1 Department of Didactics of Musical, Plastic and Corporal Expression, University of Granada, \\ 18071 Granada, Spain; felixzo@ugr.es (F.Z.O.); jjmuros@ugr.es (J.J.M.) \\ 2 Department of Didactics of Musical, Plastic and Corporal Expression, University of Almería, \\ 04120 Almería, Spain; mcastros@ual.es \\ 3 Department of Integrated Didactics, University of Huelva, 21007 Huelva, Spain \\ 4 Department of Didactics of Musical, Plastic and Corporal Expression, University of Jaén, 23071 Jaén, Spain; \\ jcachon@ujaen.es \\ 5 School of Sciences of Physical Activity, Sport and Health ECIADES, University of Santiago de Chile, \\ Santiago de Chile 9170022, Chile; cristian.cofre@usach.cl \\ 6 Sports Science and Physical Activity, University Santo Tomas, Santiago of Chile 837003, Chile \\ 7 School of Health Sciences, The University of Nottingham, Nottingham NG7 2RD, UK; \\ Emily.Knox@nottingham.ac.uk \\ * Correspondence: ramon.chacon@ddi.uhu.es; Tel.: +34-958-248-949
}

Received: 17 January 2018; Accepted: 22 February 2018; Published: 28 February 2018

\begin{abstract}
Background: Different authors have established that each type of motivation to sports can favor healthy habits in men and women, being important to promote more self-determined motivations. (2) Methods: The present study was designed to assess selected psychometric properties of the Perceived Motivational Climate in Sport Questionnaire and study the relationship between motivational climate, physical activity and gender in adolescents. Research was conducted with a sample of 2134 Spanish children (12.5 \pm 1.4 years). (3) Results: The final scale was formed of two factors. The fit of factor 1 (ego climate) was 0.867 , the fit for factor 2 was 0.851 (task climate) and the overall fit was 0.765 . Structural equation modelling identified a negative relationship between ego climate and task climate. This relationship was strong in women. A direct relationship was found between the ego climate and task climate in relation to physical activity in men and women. Nevertheless, this positive relationship was stronger in men. (4) Conclusions: PMCSQ-2 test is a good instrument to measure the motivation towards sports practice. Moreover, it is important to promote adaptive types of motivation in physical education classes in order to generate pleasure for learning and long-term physical activity engagement.
\end{abstract}

Keywords: physical activity; sport; motivation; gender

\section{Introduction}

In recent decades, physical inactivity has been identified as an important public health problem globally [1,2]. A number of initiatives aimed at encouraging more physical activity have emerged in an effort to tackle this issue [3-5]. Physical education plays an important role in promoting physical activity and healthy lifestyle behaviours. Given the associations between fostering healthy habits early in life, early life-stage development, and the lifestyle adopted throughout adulthood, physical education could be an important setting for intervention. 
In the last decades, self-determination theory and achievement goal theory have been the main theories to explain the motivational processes that operate in the behaviours of students and athletes in different contexts [6-8]. Self-determination theory posits that the motivation to enact a task is expressed along a continuum, which varies according to the degree of self-determination of the subject. Intrinsic motivation refers to the most self-determined form of motivation while amotivation refers to the least self-determined form. Extrinsic motivation falls somewhere in the middle [9]. In addition, achievement goal theory has been employed to explain motivational processes. It suggests that the goals set by individuals are largely dictated by the perception they have of their skills and principle objectives. An individual's aims tend relate to successful mastery or performance of a task $[10,11]$.

Motivation in adolescence is a key factor relating to engagement in sport. Achievement goal theory states that the way in which individuals demonstrate competence can influence their motivation to enact behaviour [11], their approach to training [12,13], or sport performance [14]. Adolescents with a task orientation are more likely to have high intrinsic motivation and engage in physical activity to cooperate with others, have fun, experience satisfaction, or strive for personal improvement [10]. This type of motivation is associated with increased long-term engagement with physical activity [15]. On the other hand, individuals with an ego orientation are more likely to set goals which relate to attaining social status, achieving recognition, or increasing economic wealth etc. All of these are related to a high risk of perceiving failure which can often lead to dropout from physical activity [16]. Achievement goal theory has been studied widely in the context of the promotion of healthy lifestyles in physical education $[17,18]$.

Recently, several studies have shown the importance of motivation in the practice of physical activity. Henriksen et al. [2] highlight the significance of this fact for developing psychosocial factors in order to avoid aggressive behaviour between peers. Annesi et al. [3] targeted social cognitive theory constructs to increase children's voluntary physical activity in school. In addition, Smith \& Petosa [19] developed a peer-mentoring approach to encourage positive motivation, leading to enhanced healthy habits relating to sport practice.

Some researchers have identified gender associations with goal orientations, in which ego orientations tend to be more developed in men and task orientation often being stronger in women [20-22]. In the context of education, a task climate is associated with positive behaviours and intrinsic motivation for learning [23]. On the other hand, an ego climate is positively related to extrinsic motivations and tends to be expressed more strongly by students who achieve higher acclaim in their high school performances [24]. Achievement goal theory has framed a large number of interventions in educational contexts [25].

In this sense, the Perceived Motivational Climate in Sport Questionnaire-2 has been widely tested to understand the motivations that operate in sport and has been validated in many contexts. For example, Newton, Duda, and Yin [26] validated this questionnaire in a sample of female athletes, concluding good internal consistency for the six-factor model underpinned by task and ego climates. Another study of interest was conducted by Smith, Cumming, and Smoll [27], who validated this instrument within a sample of young athletes. These authors reported strong factorial validity of the scale and effectively employed it in to investigate associations with anxiety and the level of enjoyment of sports practice.

This questionnaire has not only been studied in relation to sport, but also in association with physical exercise [28] and the influence exerted by coaches [12]. In the first case, Moore, Brown, and Fry [28] developed a short form of the questionnaire, obtaining good reliability for distinguishing between the two main goal orientations. This allowed development of effective strategies to improve motivation of participants. Zourbanos et al. [12] focused on the influence exerted by football coaches on the motivational climate of their players. The influence of the coach on the self-efficacy, goal orientations and empowerment of their players was validly measured. Thus, this instrument has demonstrated broad application within adult athletes, but has been insufficiently applied within people who do not practice sports frequently, individuals at an early age or to examine gender differences. 
The purpose of this study was to analyse the psychometric properties of the Perceived Motivational Climate in Sport Questionnaire-2 (PMCSQ-2) and the relationship between motivational climate and physical activity according to gender in adolescents.

\section{Materials and Methods}

\subsection{Participants and Design}

A total of 2.485 Spanish adolescents aged between 15-17 years old (15.5 \pm 1.4 years) and attending one of 20 secondary schools in Granada (Spain), were invited to participate (49.8\% male) in this research. Given the relative homogeneity of schools across Granada, a conglomerate sampling technique was used as this would enable a representative sample of adolescents from this age group from Granada $(n=19.930)$ to be recruited, assuming a sampling error of 0.02 with a $95 \%$ confidence interval. Following the conglomerate sampling approach, participants were randomly selected from 16 of the main municipalities of Granada. A total of 2.202 individuals provided informed consent and were administered the questionnaires in June 2014. Sixty eight participants were excluded for failing to produce valid questionnaires leaving a final sample of 2.134.

\subsection{Measures}

The 33-item revised Perceived Motivational Climate in Sport Questionnaire-2 (PMCSQ-2; [26]) was used to assess the motivational climate of adolescents. This questionnaire has been previously validated in Spanish physical education settings by González-Cutre, Sicilia, and Moreno [29]. Participants respond to each item on a five-point Likert scale ranging from one (strongly disagree) to five (strongly agree). The questionnaire consists of two higher order scales, with three subscales: Task Climate which incorporates Cooperative Learning, Effort/Improvement, and Important Role, and Ego Climate which incorporates Intra-team Member rivalry, Unequal Recognition, and Punishment for Mistakes. Internal consistency of the data was assessed using Cronbach's alpha and was acceptable for both perceived task and perceived ego climate subscales ( $\alpha=0.86$ and 0.85 , respectively).

To assess physical activity levels, we used the Physical Activity Questionnaire for Adolescents (PAQ-A) previously validated by Kowalski, Crocker, and Donen [30]. The instrument provides a general level of physical activity for high school students in grades 9 to 12 and aged approximately 14 to 19 years old. The PAQ-A assesses physical activity engaged in during the seven days prior to completing the questionnaire through 10 items rated on five-point Likert scales (Never = 0; Always = 5). The responses are summed to create a variable which describes physical activity levels. Respondents are asked to recall the frequency and type of physical activity they have engaged in on each of the seven days prior to completing the questionnaire. For this instrument, Cronbach's alpha obtained was $\alpha=0.82$.

\subsection{Procedure}

Permission from the Human Research ethics Committee of the University of Granada and the participating school was obtained. Informed consent was obtained from the parents or legal guardians of all participants. To be included participants had to be enrolled in secondary education in an institute of the province of Granada and to practice extracurricular physical activity. All the participants were informed that data would remain confidential. Tests were conducted during the regular physical education class with a researcher present.

\subsection{Statistical Analysis}

Data were reported as descriptive statistics (asymmetry, kurtosis, dispersion, mean and standard deviation) and were analysed using the statistical software IBM-SPSS ${ }^{\circledR}$ version 22.0 (IBM Corp, Armonk, NY, USA) for Windows. The psychometric properties of the measures were established using goodness of fit and confirmatory factor analysis using the Analysis Program FACTOR 9.3.1. [31]. 
In order to analyse the research model, a modelling approach with instructive equations at the latent variable level was applied using AMOS version 22.0. To develop the statistical model, confirmatory factor analysis was first used to check the reliability and validity of the research tool. Next relationships between all the latent variables and the categories of the model were identified.

Structural equation modelling (SEM) was used to analyse the relationships between task- and ego-involved climates and physical activity. The SEM for this data is characterised by thirty-three observed variables and two latent variables. This model makes causal explanations of the latent variables from the observed relationships between indicators. In Figure 1, the squares represent the observed variables and the circles represent the error terms. Ovals are used to indicate the latent variables.

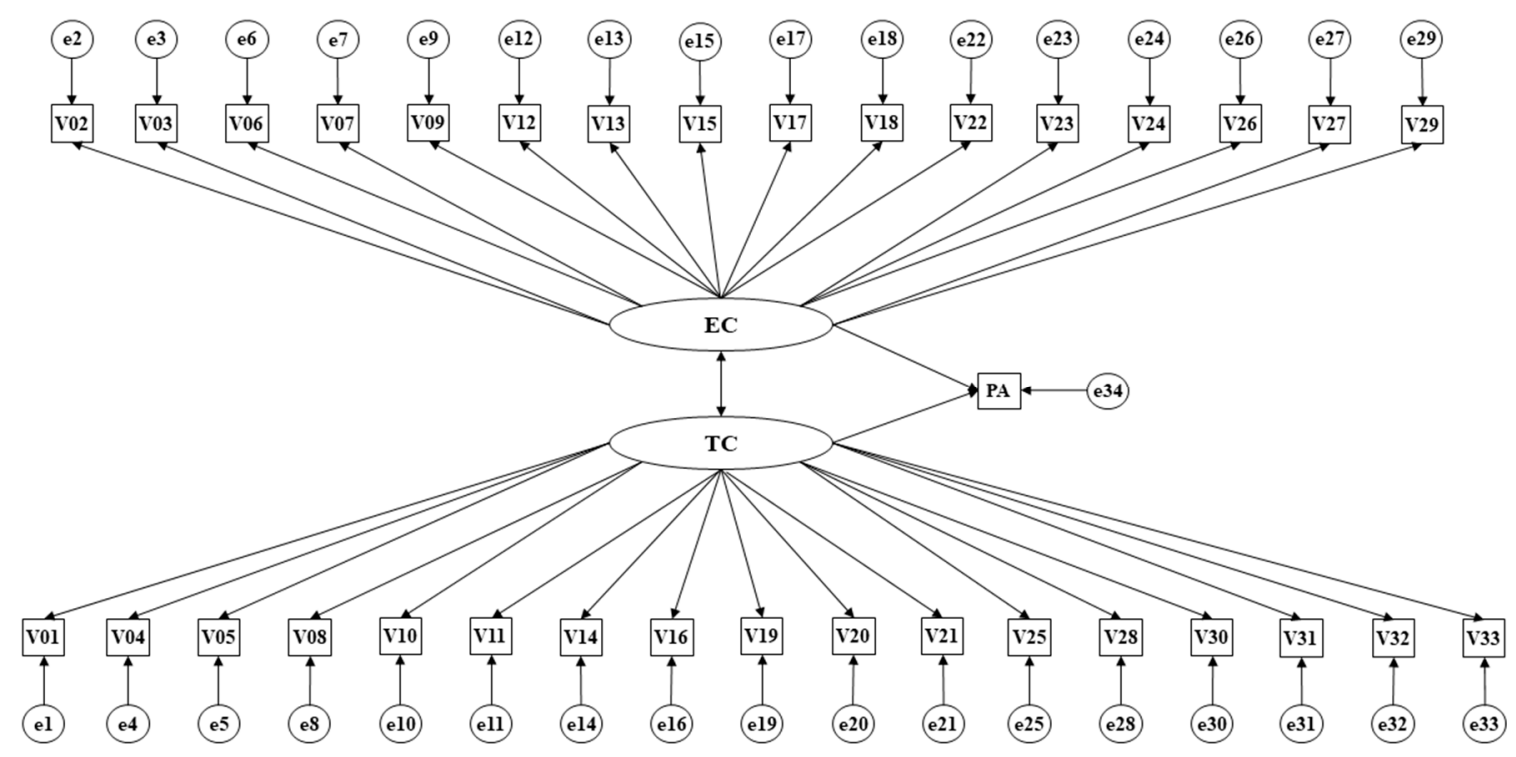

Figure 1. Model theories. Note: TC, Climate-Task; EC, Climate-Ego; PA, Physical Activity.

The task-involved climate and ego-involved climate variables are exogenous latent variables and they are each inferred using thirty-three indicators (Task: V02, V03, V06, V07, V09, V12, V13, V15, V17, V18, V22, V23, V24, V26, V27, V29; Ego: V01, V04, V05, V08, V10, V11, V14, V16, V19, V20, V21, V25, V28, V30, V31, V32, V33). Physical activity (PA) is the observed endogenous variable. The method of maximum likelihood estimation (ML) was used to estimate the parameters. We chose this method because it is consistent, unbiased and invariant to types of scale assuming that all variables have a normal distribution.

In order to verify the compatibility of the model proposed and the empirical information gathered, the model's fit was tested. The goodness of fit test was carried out on the basis of several indices. Chi-squared: non-significant values associated with $p$ indicate a good model fit. Comparative Fit Index (CFI): values higher than 0.90 indicate an acceptable model fit. Adjusted Goodness of Fit Index (AGFI): values higher than 0.90 indicate an acceptable fit. Goodness of Fit Index (GFI): values higher than 0.90 indicate an acceptable fit. Root Mean Square Error of Approximation (RMSEA): values below 0.1 indicate an acceptable model fit [32,33].

\section{Results}

Table 1 shows the descriptive findings from the PMCSQ-2 as well as the dispersion tests (asymmetry and kurtosis). After following expert recommendations [32,33], none of the variables were removed. 
Table 1. Descriptive of PMCSQ-2.

\begin{tabular}{|c|c|c|c|c|c|}
\hline & $\mathbf{M}$ & SD & $\mathbf{V}$ & A & $\mathbf{K}$ \\
\hline V01. The coach wants us to try new skills & 3.78 & 1.151 & 1.324 & -0.719 & -0.210 \\
\hline V02. The coach gets mad when a player makes a mistake & 2.62 & 1.311 & 1.720 & 0.349 & -0.993 \\
\hline V03. The coach gives most of his or her attention to the stars & 2.46 & 1.351 & 1.824 & 0.490 & -0.943 \\
\hline V04. Each person contributes in some important way & 3.42 & 1.114 & 1.242 & -0.291 & -0.515 \\
\hline V05. The coach believes that all of us are crucial to the success of the team & 3.82 & 1.189 & 1.413 & -0.745 & -0.365 \\
\hline V06. The coach praises players only when they outplay team-mates & 2.36 & 1.245 & 1.551 & 0.525 & -0.716 \\
\hline V07. The coach thinks only the starters contribute to the success of the team & 2.39 & 1.316 & 1.732 & 0.521 & -0.858 \\
\hline V08. Players feel good when they try their best & 3.93 & 1.193 & 1.423 & -0.935 & -0.059 \\
\hline V09. Players are taken out of a game for mistakes & 2.11 & 1.306 & 1.706 & 0.882 & -0.434 \\
\hline V10. Players at all skill levels have an important ro & 3.49 & 1.242 & 1.542 & -0.374 & -0.812 \\
\hline V11. Players help each other learn & 3.41 & 1.208 & 1.459 & -0.343 & -0.728 \\
\hline V12. Players a & 2.77 & 1.234 & 1.524 & 0.150 & -0.843 \\
\hline V13. The coach has his or her & 2.69 & 1.385 & 1.919 & 0.237 & -1.177 \\
\hline V14. The coach makes s & 3.55 & 1.215 & 1.475 & -0.488 & -0.644 \\
\hline V15. The coach y & 2.69 & 1.369 & 1.875 & 0.265 & -1.148 \\
\hline ssful when they improve & 3.49 & 1.132 & 1.281 & -0.476 & -0.432 \\
\hline V17. Only the players with the best stats get prai & 2.71 & 1.264 & 1.599 & 0.191 & -0.947 \\
\hline unished when they ma & 2.63 & 1.279 & 1.635 & 0.299 & -0.929 \\
\hline V19. Each player has an important role & 3.48 & 1.238 & 1.532 & -0.385 & -0.771 \\
\hline V20. Trying hard is rewarded & 3.67 & 1.230 & 1.514 & -0.610 & -0.594 \\
\hline V21. The coach encourages player & 3.69 & 1.210 & 1.463 & -0.620 & -0.534 \\
\hline co it cloar whe be or che thinlo & 2.69 & 1.281 & 1.640 & 0.212 & -0.950 \\
\hline V23. Play & 2.92 & 1.224 & 1.499 & 0.007 & -0.844 \\
\hline V24. If you & 2.66 & 1.349 & 1.819 & 0.269 & -1.099 \\
\hline V25. The $\mathrm{c}$ & 3.67 & 1.295 & 1.676 & -0.612 & -0.738 \\
\hline V26. Only the top players "get noticed" by the coach & 2.54 & 1.326 & 1.759 & 0.366 & -1.017 \\
\hline V27. Players are afraid to make mistakes & 2.89 & 1.278 & 1.634 & 0.059 & -0.977 \\
\hline V28. Players are encouraged to & 3.48 & 1.228 & 1.508 & -0.467 & -0.666 \\
\hline V29. The coach favours & 2.81 & 1.289 & 1.661 & 0.145 & -0.987 \\
\hline V30. The focus is to improve each game/practice & 3.66 & 1.174 & 1.379 & -0.508 & -0.600 \\
\hline V31.The players really "work together" as a team & 3.56 & 1.147 & 1.315 & -0.421 & -0.592 \\
\hline V32. Each player feels as if they are an important te & 3.35 & 1.179 & 1.389 & -0.236 & -0.766 \\
\hline V33. The players help each other to get better and excel & 3.44 & 1.213 & 1.471 & -0.356 & -0.735 \\
\hline
\end{tabular}

Note 1: M, Mean; SD, Standard Desviation; V, variance; A, Asymmetry; K, Kurtosis.

Table 2 shows the psychometric evaluation of the 33-item PMCSQ-2 using rotated factor matrix and load factor dimensions produced by the program FACTOR Analysis [31]. The Bartlett statistic $[18100.4(\mathrm{df}=528 ; p<0.001)]$ and Kaiser-Meyer-Olkin test $(\mathrm{KMO})=0.92$ indicate a very good fit of the data for subsequent factor analysis. The two factor solution accounted for $56.4 \%$ of the response variance. The goodness of fit index (GFI) was 0.98 and comparative fit index (CFI) was 0.96, which indicates a good fit [31]. The adjusted goodness of fit index (AGFI) produced higher values than 0.98, and the root mean square residual (RMSR) was 0.040. All these findings point to a very good fit for these items.

Table 2. Rotated Factor Matrix and Load Factor Dimensions of PMCSQ-2.

\begin{tabular}{cccccc}
\hline \multirow{2}{*}{ Variables } & \multicolumn{4}{c}{ Rotated Factor Matrix } & \multicolumn{4}{c}{ Load Factor Dimensions of PMCSQ-2 } \\
\cline { 2 - 6 } & F1 & F2 & Variables & F1 & F2 \\
\hline V01 & -0.117 & 0.365 & V02 & 0.478 & \\
V02 & 0.478 & 0.069 & V03 & 0.598 & \\
V03 & 0.598 & -0.054 & V06 & 0.504 & \\
V04 & 0.063 & 0.467 & V07 & 0.609 & \\
V05 & -0.205 & 0.492 & V09 & 0.545 & \\
V06 & 0.504 & 0.034 & V12 & 0.412 & \\
V07 & 0.609 & -0.094 & V13 & 0.611 & \\
V08 & -0.026 & 0.482 & V15 & 0.544 & \\
V09 & 0.545 & -0.123 & V17 & 0.580 & \\
V10 & 0.005 & 0.569 & V18 & 0.550 & \\
\hline
\end{tabular}


Table 2. Cont.

\begin{tabular}{cccccc}
\hline \multirow{2}{*}{ Variables } & \multicolumn{2}{c}{ Rotated Factor Matrix } & \multicolumn{3}{c}{ Load Factor Dimensions of PMCSQ-2 } \\
\cline { 2 - 6 } & $\mathbf{F 1}$ & $\mathbf{F 2}$ & Variables & F1 & F2 \\
\hline V11 & 0.012 & 0.486 & V22 & 0.548 & \\
V12 & 0.412 & 0.223 & V23 & 0.469 & \\
V13 & 0.611 & -0.012 & V24 & 0.606 & \\
V14 & -0.074 & 0.495 & V26 & 0.633 & \\
V15 & 0.544 & -0.037 & V27 & 0.392 & \\
V16 & 0.059 & 0.530 & V29 & 0.569 & \\
V17 & 0.580 & 0.038 & V01 & & 0.653 \\
V18 & 0.550 & -0.067 & V04 & & 0.467 \\
V19 & 0.037 & 0.527 & V05 & & 0.492 \\
V20 & -0.027 & 0.509 & V08 & & 0.482 \\
V21 & -0.141 & 0.548 & V10 & & 0.569 \\
V22 & 0.548 & 0.012 & V11 & & 0.486 \\
V23 & 0.469 & 0.212 & V14 & & 0.495 \\
V24 & 0.606 & -0.031 & V16 & & 0.530 \\
V25 & -0.025 & 0.464 & V19 & & 0.527 \\
V26 & 0.633 & -0.071 & V20 & & 0.509 \\
V27 & 0.392 & 0.138 & V21 & & 0.548 \\
V28 & -0.017 & 0.482 & V25 & & 0.464 \\
V29 & 0.569 & -0.010 & V28 & & 0.482 \\
V30 & 0.093 & 0.465 & V30 & & 0.465 \\
V31 & 0.035 & 0.536 & V31 & & 0.536 \\
V32 & 0.076 & 0.519 & V32 & & 0.519 \\
V33 & 0.123 & 0.563 & V33 & & 0.563 \\
\hline & & & Alpha (0.765) & 0.867 & 0.851 \\
\hline
\end{tabular}

The final scale was formed of two factors (F1 and F2). The ego climate (F1) comprised sixteen variables, and the task climate (F2) comprised seventeen variables. The overall measure demonstrated good fit $(\alpha=0.765)$, consisting of 0.867 for factor 1 (ego climate) and 0.851 for factor 2 (task climate).

A structural equation model was designed in order to establish relationships between motivation, physical activity and gender. The first model (Figure 2) analysed motivational climate in men. The chi-square test statistic was not significant at $.05\left(\chi^{2}=99.330 ; \mathrm{gl}=12 ; p<0.001\right)$, which suggests model fit to be acceptable. This statistic has no upper limit which cannot be interpreted in a standardised way and may also be sensitive to sample size. Other standardised rates are less sensitive to sample size (Jöreskog and Sörbom [34]). The comparative fix index (CFI) was 0.955 and the incremental fit index (IFI) was 0.960, which indicates good fit [31]. Finally, the normalised fit index (NFI) was 0.960 and the root mean square error of approximation (RMSEA) was less than 0.08 which indicates acceptable fit. In general, a good fit of the model to empirical data was observed.

Table 3 shows the estimated parameter values for men. Statistically significant relationships at the level of 0.001 were identified between all variables in men. Positive relationships were found between Task Climate and its items, as well as between Ego Climate and its items. In fact, when the critical ration was considered, these associations were especially strong. Furthermore, Task Climate was positively associated with physical activity. Similarly, Ego Climate revealed a direct relationship with engagement in physical activity, although this relationship was weaker than for Task Climate. A negative relationship was observed between Task Climate and Ego Climate.

The second model (Figure 3 ) analysed motivational climate in women. The chi-square test statistic was not significant at $0.05\left(\chi^{2}=77.801 ; \mathrm{gl}=12 ; p<0.001\right)$, which suggests that model fit is acceptable. The comparative fix index (CFI) was 0.971 and the incremental fit index (IFI) was 0.971 , which indicates a good fit [31]. Finally, the normalised fit index (NFI) was 0.966 and the root mean square error of approximation (RMSEA) was 0.072 which indicates an acceptable fit. In general, a good fit of the model to empirical data was observed. 


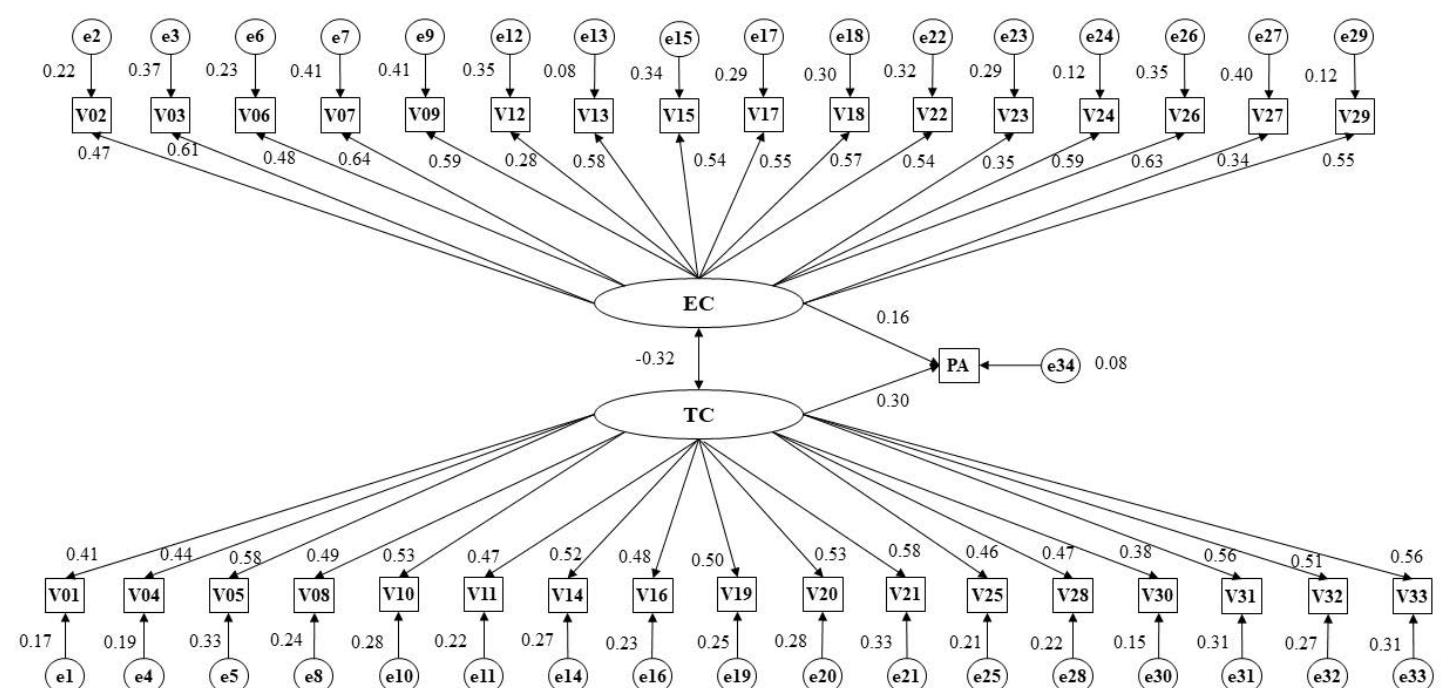

Figure 2. Multigroup structural equation model: men. Note: TC, Climate-Task; EC, Climate-Ego; PA, Physical Activity.

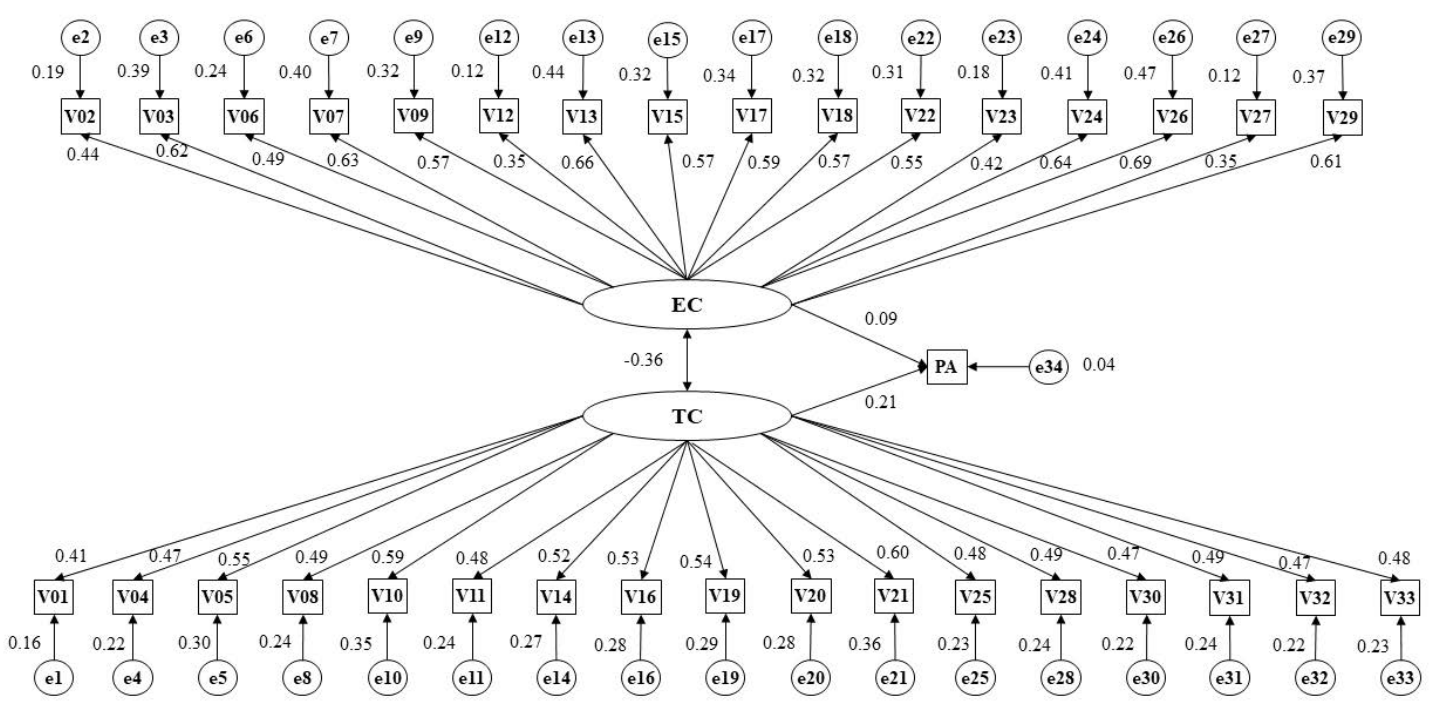

Figure 3. Multigroup structural equation model: women. Note: TC, Climate-Task; EC, Climate-Ego; PA, Physical Activity.

Table 4 shows the estimated parameter values for women. Statistically significant relationships at the level of 0.001 were found between all variables except between Ego Climate and physical activity $(p<0.01)$. As for men, a positive relationship was identified between Task Climate and its dimensions, as well as between Ego Climate and its dimensions. The main associations were weaker in women than men between Task Climate and physical activity ( $r=0.234$ vs. 0.311$)$. Similarly, the associations between Ego Climate and physical activity engagement were weaker in women ( $r=0.095$ vs. $r=0.155)$. Finally, the negative relationship between Task Climate and Ego Climate was stronger in women $(r=-0.343$ vs. $r=0.306)$. 
Table 3. Weights and standardized regression weights in men.

\begin{tabular}{|c|c|c|c|c|c|c|c|}
\hline \multirow{2}{*}{\multicolumn{3}{|c|}{ Relations between Variables }} & \multicolumn{4}{|c|}{ R.W. } & \multirow{3}{*}{$\begin{array}{c}\text { S.R.W. } \\
\text { Estimates } \\
0.467\end{array}$} \\
\hline & & & \multirow{2}{*}{$\begin{array}{c}\text { Estimate } \\
1.000\end{array}$} & \multirow{2}{*}{$\begin{array}{c}\text { S.E. } \\
-\end{array}$} & \multirow{2}{*}{$\begin{array}{c}\text { C.R. } \\
-\end{array}$} & \multirow{2}{*}{$\begin{array}{l}P \\
-\end{array}$} & \\
\hline V02 & $\leftarrow$ & $\mathrm{CE}$ & & & & & \\
\hline V03 & $\leftarrow$ & $\mathrm{CE}$ & 1.365 & 0.104 & 13.110 & $* * *$ & 0.608 \\
\hline V06 & $\leftarrow$ & $\mathrm{CE}$ & 1.012 & 0.089 & 11.429 & $* * *$ & 0.476 \\
\hline V07 & $\leftarrow$ & $\mathrm{CE}$ & 1.405 & 0.104 & 13.480 & $* * *$ & 0.643 \\
\hline V09 & $\leftarrow$ & $\mathrm{CE}$ & 1.310 & 0.101 & 12.916 & $* * *$ & 0.590 \\
\hline V12 & $\leftarrow$ & $\mathrm{CE}$ & 0.566 & 0.074 & 7.672 & $* * *$ & 0.277 \\
\hline V13 & $\leftarrow$ & $\mathrm{CE}$ & 1.341 & 0.105 & 12.788 & $* * *$ & 0.579 \\
\hline V15 & $\leftarrow$ & $\mathrm{CE}$ & 1.212 & 0.098 & 12.314 & $* * *$ & 0.540 \\
\hline V17 & $\leftarrow$ & $\mathrm{CE}$ & 1.132 & 0.091 & 12.380 & $* * *$ & 0.545 \\
\hline V18 & $\leftarrow$ & $\mathrm{CE}$ & 1.166 & 0.092 & 12.661 & $* * *$ & 0.568 \\
\hline V22 & $\leftarrow$ & $\mathrm{CE}$ & 1.155 & 0.093 & 12.352 & $* * *$ & 0.543 \\
\hline V23 & $\leftarrow$ & $\mathrm{CE}$ & 0.704 & 0.077 & 9.195 & $* * *$ & 0.348 \\
\hline V24 & $\leftarrow$ & $\mathrm{CE}$ & 1.295 & 0.100 & 12.898 & $* * *$ & 0.589 \\
\hline V26 & $\leftarrow$ & $\mathrm{CE}$ & 1.394 & 0.104 & 13.384 & $* * *$ & 0.634 \\
\hline V27 & $\leftarrow$ & $\mathrm{CE}$ & 0.719 & 0.079 & 9.101 & $* * *$ & 0.343 \\
\hline V29 & $\leftarrow$ & $\mathrm{CE}$ & 1.164 & 0.094 & 12.389 & $* * *$ & 0.546 \\
\hline V01 & $\leftarrow$ & $\mathrm{CT}$ & 1.000 & - & - & - & 0.410 \\
\hline V04 & $\leftarrow$ & $\mathrm{CT}$ & 1.028 & 0.104 & 9.875 & $* * *$ & 0.436 \\
\hline V05 & $\leftarrow$ & $\mathrm{CT}$ & 1.424 & 0.125 & 11.347 & $* * *$ & 0.577 \\
\hline V08 & $\leftarrow$ & $\mathrm{CT}$ & 1.251 & 0.120 & 10.464 & $* * *$ & 0.485 \\
\hline V10 & $\leftarrow$ & $\mathrm{CT}$ & 1.388 & 0.127 & 10.952 & $* * *$ & 0.533 \\
\hline V11 & $\leftarrow$ & $\mathrm{CT}$ & 1.208 & 0.117 & 10.315 & $* * *$ & 0.472 \\
\hline V14 & $\leftarrow$ & $\mathrm{CT}$ & 1.328 & 0.123 & 10.832 & $* * *$ & 0.521 \\
\hline V16 & $\leftarrow$ & $\mathrm{CT}$ & 1.177 & 0.113 & 10.444 & $* * *$ & 0.483 \\
\hline V19 & $\leftarrow$ & $\mathrm{CT}$ & 1.271 & 0.120 & 10.636 & $* * *$ & 0.501 \\
\hline V20 & $\leftarrow$ & $\mathrm{CT}$ & 1.368 & 0.126 & 10.881 & $* * *$ & 0.526 \\
\hline V21 & $\leftarrow$ & $\mathrm{CT}$ & 1.448 & 0.128 & 11.353 & $* * *$ & 0.578 \\
\hline V25 & $\leftarrow$ & $\mathrm{CT}$ & 1.243 & 0.122 & 10.155 & $* * *$ & 0.458 \\
\hline V28 & $\leftarrow$ & $\mathrm{CT}$ & 1.229 & 0.119 & 10.284 & $* * *$ & 0.469 \\
\hline V30 & $\leftarrow$ & $\mathrm{CT}$ & 0.956 & 0.104 & 9.174 & $* * *$ & 0.385 \\
\hline V31 & $\leftarrow$ & $\mathrm{CT}$ & 1.337 & 0.120 & 11.172 & $* * *$ & 0.557 \\
\hline V32 & $\leftarrow$ & $\mathrm{CT}$ & 1.276 & 0.118 & 10.775 & $* * *$ & 0.515 \\
\hline V33 & $\leftarrow$ & $\mathrm{CT}$ & 1.451 & 0.130 & 11.172 & $* * *$ & 0.557 \\
\hline PA & $\leftarrow$ & $\mathrm{CE}$ & 0.200 & 0.045 & 4.417 & $* * *$ & 0.157 \\
\hline PA & $\leftarrow$ & $\mathrm{CT}$ & 0.483 & 0.066 & 7.315 & $* * *$ & 0.298 \\
\hline $\mathrm{CT}$ & $\leftrightarrow$ & $\mathrm{CE}$ & -0.090 & 0.013 & -6.659 & $* * *$ & -0.319 \\
\hline
\end{tabular}

Note 1: R.W., Regression Weights; S.R.W., Standardized Regression Weights; S.E., Estimation of Error; C.R., Critical Ratio. Note 2: TC, Task Climate; EC, Ego Climate; PA, Physical Activity. Note 3: ${ }^{* * *} p<0.001$. Note 4: $\leftarrow$, Lines of influence between the latent and observable indicators; Note 5: $\leftrightarrow$, Lines of influence between the latent indicators.

Table 4. Weights and standardized regression weights in women.

\begin{tabular}{lllccccc}
\hline \multirow{2}{*}{ Relations between Variables } & \multicolumn{5}{c}{ R.W. } \\
\cline { 3 - 7 } & & Estimate & S.E. & C.R. & P & Estimates \\
\hline V02 & $\leftarrow$ & CE & 1.000 & - & - & - & 0.437 \\
V03 & $\leftarrow$ & CE & 1.440 & 0.113 & 12.707 & $* * *$ & 0.622 \\
V06 & $\leftarrow$ & CE & 1.006 & 0.089 & 11.261 & $* * *$ & 0.488 \\
V07 & $\leftarrow$ & CE & 1.419 & 0.111 & 12.784 & $* * *$ & 0.631 \\
V09 & $\leftarrow$ & CE & 1.236 & 0.101 & 12.185 & $* * *$ & 0.568 \\
V12 & $\leftarrow$ & CE & 0.744 & 0.081 & 9.161 & $* * *$ & 0.353 \\
V13 & $\leftarrow$ & CE & 1.559 & 0.119 & 13.049 & $* * *$ & 0.663 \\
V15 & $\leftarrow$ & CE & 1.353 & 0.111 & 12.194 & $* * *$ & 0.569 \\
V17 & $\leftarrow$ & CE & 1.282 & 0.104 & 12.373 & $* * *$ & 0.587 \\
V18 & $\leftarrow$ & CE & 1.285 & 0.105 & 12.202 & $* * *$ & 0.570 \\
V22 & $\leftarrow$ & CE & 1.213 & 0.101 & 12.027 & $* * *$ & 0.553 \\
\hline
\end{tabular}


Table 4. Cont.

\begin{tabular}{|c|c|c|c|c|c|c|c|}
\hline \multirow{2}{*}{\multicolumn{3}{|c|}{ Relations between Variables }} & \multicolumn{4}{|c|}{ R.W. } & \multirow{3}{*}{$\begin{array}{c}\text { S.R.W. } \\
\text { Estimates } \\
0.423\end{array}$} \\
\hline & & & \multirow{2}{*}{$\begin{array}{c}\text { Estimate } \\
0.890\end{array}$} & \multirow{2}{*}{$\begin{array}{c}\text { S.E. } \\
0.086\end{array}$} & \multirow{2}{*}{$\begin{array}{c}\text { C.R. } \\
10.344\end{array}$} & \multirow{2}{*}{$\frac{\mathbf{P}}{* * *}$} & \\
\hline V23 & $\leftarrow$ & $\mathrm{CE}$ & & & & & \\
\hline V24 & $\leftarrow$ & $\mathrm{CE}$ & 1.504 & 0.117 & 12.873 & $* * *$ & 0.641 \\
\hline V26 & $\leftarrow$ & CE & 1.561 & 0.118 & 13.239 & $* * *$ & 0.687 \\
\hline V27 & $\leftarrow$ & $\mathrm{CE}$ & 0.768 & 0.085 & 9.034 & $* * *$ & 0.346 \\
\hline V29 & $\leftarrow$ & $\mathrm{CE}$ & 1.346 & 0.107 & 12.566 & $* * *$ & 0.607 \\
\hline V01 & $\leftarrow$ & $\mathrm{CT}$ & 1.000 & - & - & - & 0.406 \\
\hline V04 & $\leftarrow$ & $\mathrm{CT}$ & 1.114 & 0.109 & 10.248 & $* * *$ & 0.466 \\
\hline V05 & $\leftarrow$ & $\mathrm{CT}$ & 1.430 & 0.129 & 11.107 & $* * *$ & 0.551 \\
\hline V08 & $\leftarrow$ & $\mathrm{CT}$ & 1.207 & 0.115 & 10.460 & $* * *$ & 0.485 \\
\hline V10 & $\leftarrow$ & $\mathrm{CT}$ & 1.585 & 0.139 & 11.443 & $* * *$ & 0.591 \\
\hline V11 & $\leftarrow$ & $\mathrm{CT}$ & 1.252 & 0.120 & 10.458 & $* * *$ & 0.485 \\
\hline V14 & $\leftarrow$ & $\mathrm{CT}$ & 1.354 & 0.126 & 10.783 & $* * *$ & 0.516 \\
\hline V16 & $\leftarrow$ & $\mathrm{CT}$ & 1.260 & 0.116 & 10.895 & $* * *$ & 0.528 \\
\hline V19 & $\leftarrow$ & $\mathrm{CT}$ & 1.465 & 0.134 & 10.970 & $* * *$ & 0.536 \\
\hline V20 & $\leftarrow$ & $\mathrm{CT}$ & 1.393 & 0.128 & 10.895 & $* * *$ & 0.528 \\
\hline V21 & $\leftarrow$ & $\mathrm{CT}$ & 1.594 & 0.138 & 11.533 & $* * *$ & 0.603 \\
\hline V25 & $\leftarrow$ & $\mathrm{CT}$ & 1.335 & 0.129 & 10.371 & $* * *$ & 0.477 \\
\hline V28 & $\leftarrow$ & $\mathrm{CT}$ & 1.289 & 0.122 & 10.560 & $* * *$ & 0.494 \\
\hline V30 & $\leftarrow$ & $\mathrm{CT}$ & 1.185 & 0.115 & 10.295 & $* * *$ & 0.470 \\
\hline V31 & $\leftarrow$ & $\mathrm{CT}$ & 1.203 & 0.115 & 10.468 & $* * *$ & 0.486 \\
\hline V32 & $\leftarrow$ & $\mathrm{CT}$ & 1.201 & 0.116 & 10.321 & $* * *$ & 0.473 \\
\hline V33 & $\leftarrow$ & $\mathrm{CT}$ & 1.220 & 0.118 & 10.362 & $* * *$ & 0.476 \\
\hline PA & $\leftarrow$ & $\mathrm{CE}$ & 0.111 & 0.046 & 2.427 & 0.015 & 0.086 \\
\hline PA & $\leftarrow$ & $\mathrm{CT}$ & 0.337 & 0.063 & 5.391 & $* * *$ & 0.208 \\
\hline $\mathrm{CT}$ & $\leftrightarrow$ & $\mathrm{CE}$ & -0.098 & 0.014 & -7.033 & $* * *$ & -0.359 \\
\hline
\end{tabular}

Note 1: R.W., Regression Weights; S.R.W., Standardized Regression Weights; S.E., Estimation of Error; C.R., Critical Ratio. Note 2: TC, Task Climate; EC, Ego Climate; PA, Physical Activity. Note 3: ${ }^{* * *} p<0.001$. Note 4: $\leftarrow$, Lines of influence between the latent and observable indicators; Note $5: \leftrightarrow$, Lines of influence between the latent indicators.

\section{Discussion}

The purpose of the present study was to examine the structure and psychometric properties of the PMCSQ-2. A further aim was to examine the relationship between motivational climate and physical activity according to gender in a large sample of Spanish high school students enrolled in physical education classes.

Findings supported the validity of the instrument in establishing the motivational climate perceived by adolescent students in the context of physical activity. Cronbach's alpha of a two-dimensional model was satisfactory. The period of adolescence is considered to be essential for human development and behaviour as patterns of behaviour followed during this stage are often repeated in adulthood. It is therefore a vital period for developing adaptive types of motivation towards physical activity [35]. Adolescents with a greater intrinsically directed predisposition towards physical activity and sport, who engage for hedonistic reasons are more likely to experience repeated satisfaction and pleasure from their engagement and continue to engage in the future $[15,36,37]$.

The present results loaded consistently on two factors suggesting the presence of two stable dimensions (ego climate and task climate). The item has been used previously to demonstrate the cognitive, emotional and behavioural patterns of adolescent students in the context of sport [38]. Achievement goal theory has been applied successfully in other contexts also, with task-related constructs demonstrating positive relationships with homework tasks, learning and personal development. Further, ego orientation and social comparisons have demonstrated negative implications during adolescence $[19,39]$.

Students who are task involved, meaning that they strive for personal improvement and engage for self-regulated reasons, often perceive physical activity as a means of experiencing enjoyment and 
challenging themselves. They tend to focus more on the process rather than the end result. On the other hand, those who are ego-involved often perceive physical activity as a way to raise their status, obtain extrinsic rewards, or to achieve success with minimum effort [6]. The results are similar to those of Grasten [40], who also found that girls tended to report higher proportions of task orientation relative to ego orientation than boys. This resulted in them prioritising the learning process and effort, showing persistence and adhering to physical activity [41].

The structural equation model developed demonstrated a negative relationship between ego climate and task climate. Typically, this relationship is weaker in females who have also traditionally shown lower levels of ego involvement [42,43]. In addition, previous studies have suggested that males typically experience greater extrinsic motivations towards sport and experience stronger rivalries and social comparisons $[14,40,41]$. The present results also found a direct relationship between perception of an ego climate and perception of a task climate in the context of physical activity in both males and females. However, in the present study, males did not exhibit stronger relationships than females. As the present research was conducted in an academic as opposed to a sport setting, this could partially explain these differences. Indeed, previous research by Moreno-Murcia et al. [44] similarly found that females exhibited a greater negative association between task and ego orientation. This is possibly due to females elevated levels of task orientation in academic environments exerting a protective effect over the potentially damaging ego orientation [45].

This study has two main contributions to the literature. The first contribution relates to the importance of motivation for promoting pleasure in learning and long-term physical activity practice. For instance, educational interventions should be grounded in self-improvement and cooperation in order to set a motivational climate that helps to develop positive experiences in physical education. The second contribution is associated with the importance of promoting adaptive motivational patterns for learning within different individuals in order to enhance their adherence to physical activity. Teachers who create ego orientations at school could generate negative effects for students $[8,46]$.

The present research is novel in that it examines the reliability of the PMCSQ-2 within a previously unexamined sample. Young adolescents who do not participate in high-level organized sport present a large and important demographic to consider in the context of behaviour change and public health. The present results will enable the future design of better research studies within this population.

It is important to acknowledge the main limitations of this study. The present research is limited by its descriptive cross-sectional design, which precludes conclusions on causality or directionality from being made. In addition, another limitation is the differentiation of the two basic dimensions of motivational climate -task climate and ego climate- without considering the three factors which constitute each one. The present research sought to identify the main goal orientations of adolescents who were not athletes, full consideration of the six factors would provide greater insight.

Future investigations should try to establish links between students' perceived class climate and students' achievement goals. It would also be interesting to examine other age groups such as, younger schoolchildren or university students. Secondly, other interacting variables should be examined such as the wider social context or the individual's educational level. In summary, the results of the present study support the use of PMCSQ-2 as a valid and reliable measure of motivation towards sport in youth populations.

\section{Conclusions}

The PMCSQ-2 demonstrates strong psychometric properties and provides a reliable measure of perceived motivational climates in adolescent males and females. An indirect relationship exists between task-involved orientation and ego-involved orientation which is stronger in females than in males. Further, both types of orientation positively related to physical activity, but this relationship was stronger in individuals who perceived a greater task climate. It is therefore important to promote task-related motivations in order to develop pleasure in learning and encourage long-term physical activity practice. 
Acknowledgments: No external funding was secured for this study.

Author Contributions: Félix Zurita Ortega and José Joaquín Muros conceived the hypothesis of this study. Félix Zurita Ortega, Manuel Castro Sánchez, Javier Cachón Zagalaz and Cristian Cofré Bolados participated in data collection. Ramón Chacón Cuberos, Félix Zurita Ortega y Emily Knox analysed the data. All authors contributed to data interpretation of statistical analysis. Ramón Chacón Cuberos, José Joaquín Muros and Emily Knox wrote the paper with significant input from Félix Zurita Ortega. All authors read and approved the final manuscript.

Conflicts of Interest: The authors declare no conflict of interest.

\section{References}

1. Pratt, M.; Norris, J.; Lobelo, F.; Roux, L.; Wang, G. The cost of physical inactivity: Moving the 21st century. Br. J. Sport Med. 2014, 48, 171-173. [CrossRef] [PubMed]

2. Henriksen, P.W.; Rayce, S.B.; Melkevik, O.; Due, P.; Holstein, B.E. Social background, bullying and physical inactivity. National study of 11 to 15 year olds. Scand. J. Med. Sci. Sports 2016, 26, 1249-1255. [CrossRef] [PubMed]

3. Annesi, J.; Walsh, S.; Greenwood, B. Increasing children's voluntary physical activity outside of school hours through targeting social cognitive theory variables. J. Prim. Care Commun. Health 2016, 7, 234-241. [CrossRef] [PubMed]

4. Brusseau, T.A.; Hannon, J.; Burns, R. The effect of a comprehensive school physical activity program on physical activity and health-related fitness in children from low-income families. J. Phys. Act Health 2016, 13, 888-894. [CrossRef] [PubMed]

5. Smith, A.L.; Gustafsson, H.; Hassmén, P. Peer motivational climate and burnout perceptions of adolescent athletes. Psychol. Sport Exerc. 2010, 11, 453-460. [CrossRef]

6. Atkins, M.; Johnson, D.; Force, E.; Petrie, T. Peers, parents and coaches, oh my! The relation of the motivational climate to boys' intention to continue in sport. Psychol. Sport Exerc. 2015, 16, 170-180. [CrossRef]

7. Ruiz, M.C.; Haapanen, S.; Tolvanen, A.; Robazza, C.; Duda, J.L. Predicting athletes' functional and dysfunctional emotions: The role of the motivational climate and motivational regulations. J. Sports Sci. 2016, 26, 1-9. [CrossRef] [PubMed]

8. Chacón, R.; Zurita, F.; Castro, M.; Espejo, T.; Martínez, A.; Pérez, A.J. Motivational climate in sport and its relationship with digital sedentary leisure habits in university students. Saude Soc. 2017, 26, 29-39. [CrossRef]

9. Ryan, R.; Deci, E.L. Self-determination theory and the facilitation of intrinsic motivation, social development, and well-being. Am. Psychol. 2000, 55, 68-78. [CrossRef] [PubMed]

10. Gonçalves, C.E.; Coelho, M.J.; Cruz, J.; Torregrosa, M.; Cumming, S. The effect of achievement goals on moral attitudes in young athletes. J. Sports Sci. Med. 2010, 9, 605-611. [PubMed]

11. Nicholls, J.G. The Competitive Ethos and Democratic Education; Harvard University Press: Cambridge, UK, 1989; pp. 99-135.

12. Zourbanos, N.; Haznadar, A.; Papaioannou, A.; Tzioumakis, Y.; Krommidas, C.; Hatzigeorgiadis, A. The Relationships between Athletes' Perceptions of Coach-Created Motivational Climate, Self-Talk, and Self-Efficacy in Youth Soccer. J. Appl. Sport Psychol. 2015, 28, 97-112. [CrossRef]

13. Sánchez-Jover, F.; Gómez, A. Training habits, motivation, quality of life and sport injuries in 12 to 15 years old basketball players. J. Hum. Sport Exec. 2017, 12, 760-774. [CrossRef]

14. Zurita, F.; Muros, J.J.; Rodríguez, S.; Zafra, E.; Knox, E.; Castro, M. Associations of motivation, self-concept and resilience with the competitive level of Chilean judokas. Arch Budo 2016, 12, 201-209.

15. Jaakkola, T.; Ntoumanis, N.; Liukkonen, J. Motivational climate, goal orientation, perceived sport ability, and enjoyment within Finnish junior ice hockey players. Scand. J. Med. Sci. Sport 2016, 26, 109-115. [CrossRef] [PubMed]

16. Van den Berghe, L.; Soenens, B.; Vansteenkiste, M.; Aelterman, N.; Cardon, G.; Tallir, I.B.; Haerens, L. Observed need-supportive and need-thwarthing teaching behavior in physical education: Do teachers' motivational orientations matter? Psychol. Sport Exerc. 2013, 14, 650-661. [CrossRef]

17. Fernández-Rio, J.; Méndez-Giménez, A.; Cecchini Estrada, J.A. A cluster analysis on students' perceived motivational climate. Implications on psycho-social variables. Span. J. Psychol. 2014, 17, E18. [CrossRef] [PubMed] 
18. Wang, J.C.; Liu, W.C.; Chatzisarantis, N.L.; Lim, C.B. Influence of Perceived Motivational Climate on Achievement Goals in Physical Education: A Structural Equation Mixture Modeling Analysis. J. Sport Exerc. Psychol. 2010, 32, 324-338. [CrossRef] [PubMed]

19. Smith, L.; Petosa, R. A structured peer-mentoring method for Physical Activity behavior change among adolescents. J. School Nurs. 2016, 32, 315-323. [CrossRef] [PubMed]

20. Litalien, D.; Morin, A.J.; Mclnerney, D.M. Achievement goal profile among adolescent males and females. Dev. Psychol. 2017, 53, 731-751. [CrossRef] [PubMed]

21. Kool, A.; Mainhard, T.; Brekelmans, M.; van Beukelen, P.; Jaarsma, D. Goal orientations of health profession students throughout the undergraduate program: A multilevel study. BMC Med. Educ. 2016, 16, 100. [CrossRef] [PubMed]

22. Erturan-Ilker, G.; Yu, C.; Alemdaroğlu, U.; Köklü, Y. Basic psychological needs and self-determined motivation in PE to predict health-related fitness level. J. Sport Health Res. 2018, 10, 91-100.

23. Keegan, R.J.; Harwood, C.G.; Spray, C.M.; Lavallee, D. A qualitative investigation of the motivational climate in elite sport. Psychol. Sport Exerc. 2014, 15, 97-107. [CrossRef]

24. Zhang, T.; Solmon, M.A.; Kosma, M.; Carson, R.L.; Gu, X. Need support, need satisfaction, intrinsic motivation, and physical activity participation among middle school students. J. Teach. Phys. Educ. 2011, 30, 51-68. [CrossRef]

25. Hastie, P.; Sinelnikov, O.; Walhead, T.; Layne, T. Perceived and actual motivational climate of a mastery-involving sport education seasons. Eur. Phys. Educ. Rev. 2014, 20, 215-228. [CrossRef]

26. Newton, M.; Duda, J.; Yin, Z. Examination of the psychometric properties of the Perceived Motivational Climate in Sport Questionnaire-2 in a sample of female athletes. J. Sports Sci. 2000, 18, 275-290. [CrossRef] [PubMed]

27. Smith, R.; Cumming, S.P.; Smoll, F.L. Development and Validation of the Motivational Climate Scale for Youth Sports. J. Appl. Sport Psychol. 2008, 20, 116-136. [CrossRef]

28. Moore, E.W.; Brown, T.C.; Fry, M.D. Psychometric Properties of the Abbreviated Perceived Motivational Climate in Exercise Questionnaire. Meas. Phys. Educ. Exerc. Sci. 2015, 19, 186-199. [CrossRef]

29. González-Cutre, D.; Sicilia, A.; Moreno, J.A. The social-cognitive model of achievement motivation in physical education. Psicothema 2008, 20, 642-651.

30. Kowalski, K.C.; Crocker, P.R.; Donen, R.M. The physical activity questionnaire for older children (PAQ-C) and adolescents (PAQ-A) manual. Coll. Kinesiol. 2004, 87, 1-38.

31. Lorenzo-Seva, U.; Ferrando, P.J. FACTOR: A computer program to fit the exploratory factor analysis model. Behav. Res. Methods 2006, 38, 88-91. [CrossRef] [PubMed]

32. Schmider, E.; Ziegler, M.; Danay, E.; Beyer, L.; Bühner, M. Is it really robust? Reinvestigating the robustness of ANOVA against violations of the normal distribution assumption. Eur. Res. J. Methods Behav. Soc. Sci. 2010, 6, 147-151. [CrossRef]

33. Hu, L.T.; Bentler, P.M. Fit indices in covariance structure modeling: Sensitivity to underparameterized model misspecification. Psychol. Methods 1988, 3, 424-453. [CrossRef]

34. Jöreskog, K.G.; Sörbom, D. LISREL 8: Structural Equation Modeling with the SIMPLIS Command Language; SSI: Lincolnwood, IL, USA, 1993; pp. 123-135.

35. Wang, M.T.; Chow, A.; Amemiya, J. Who Wants to Play? Sport Motivation Trajectories, Sport Participation, and the Development of Depressive Symptoms. J. Youth Adolesc. 2017, 1-17. [CrossRef] [PubMed]

36. Martins, J.; Marques, A.; Sarmento, H.; Carreiro, F. Adolescents' perspectives on the barriers and facilitators of physical activity: A systematic review of qualitative studies. Health Educ. Res. 2015, 30, 742-755. [CrossRef] [PubMed]

37. Joesaar, H.; Hein, V.; Hagger, M.S. Youth athletes' perception of autonomy support from the coach, peer motivational climate and intrinsic motivation in sport setting: One-year effects. Psychol. Sport Exerc. 2012, 13, 257-262. [CrossRef]

38. Papaioannou, A.; Ampatzoglou, G.; Kalogiannis, P.; Sagovits, A. Social agents, achievement goals, satisfaction and academic achievement in youth sport. Psychol. Sport Exerc. 2008, 9, 122-141. [CrossRef]

39. López, G.F.; Ahmed, D.; Díaz, A. Level of habitual physical activity among 13-year-old adolescents from Spain and India. A cross-cultural study. Sport TK 2017, 6, 67-74. 
40. Grasten, A. Testing the model of motivational climate, goal orientations, expectancy beliefs, task values in school physical education, and associated links to light- to vigorous-intensity physical activity. Int. J. Sport Psychol. 2016, 47, 408-427. [CrossRef]

41. Curran, T.; Hill, A.P.; Hall, H.K.; Jowett, G.E. Relationships between the coach-created motivational climate and athlete engagement in youth sport. J. Sport Exerc. Psychol. 2015, 7, 193-198. [CrossRef] [PubMed]

42. González-Valero, G.; Zurita-Ortega, F.; Martínez-Martínez, A. Motivational and physical activity outlook in students: A systematic review. Educ. Sport Health Phys. Act. 2017, 1, 41-58. [CrossRef]

43. Harwood, C.G.; Keegan, R.J.; Smith, J.M.; Raine, A.S. A systematic review of the intrapersonal correlates of motivational climate perceptions in sport and physical activity. Psychol. Sport Exerc. 2015, 18, 9-25. [CrossRef]

44. Moreno-Murcia, J.A.; Sicilia, A.; Cervelló, E.; Huéscar, E.; Dumitru, D.C. The relationship between goal orientations, motivational climate and selfreported discipline in physical education. J. Sports Sci. Med. 2011, 10, 119-129. [PubMed]

45. Bakirtzoglou, P.; Loannou, P. Goal orientations, motivational climate and ispositional flow in Greek secondary education students participating in physical education lesson: Differences based on gender. Facta Univ. Ser. Phys. Educ. Sport 2011, 9, 295-306.

46. Breiger, J.; Cumming, S.P.; Smith, R.E.; Smoll, F. Winning, motivational climate, and young athletes' competitive experiences: Some notable sex differences. Int. J. Sports Sci. Coach. 2015, 10, 395-411. [CrossRef]

(C) 2018 by the authors. Licensee MDPI, Basel, Switzerland. This article is an open access article distributed under the terms and conditions of the Creative Commons Attribution (CC BY) license (http://creativecommons.org/licenses/by/4.0/). 